\title{
Feasibility of Using Infant Testing during Immunization to Estimate Mother-to-Child- Transmission Rates in Zambia
}

\author{
CURRENT STATUS: UNDER REVIEW \\ BMC Infectious Diseases $\triangle$ BMC Series \\ Joseph Simbaya \\ ఐ j.simbaya@unza.zmCorresponding Author \\ ORCiD: https://orcid.org/0000-0001-7518-6370 \\ Patricia Funjika \\ University of Zambia, Institute of Economic and Social Research \\ Arthur Moonga \\ University of Zambia Institute of Economic and Social Research \\ John Mwale \\ National AIDS Council \\ Chipepo Kankasa \\ University Teaching Hospital, Pediatric HIV Centre of Excellence
}

DOI:

10.21203/rs.3.rs-17305/v1

\section{SUBJECT AREAS}

Infectious Diseases

\section{KEYWORDS}

HIV, Mother-to-child transmission, Africa, Children 
Abstract

Background: This study piloted the feasibility of infant testing in immunization services as a strategy for estimating MTCT rates among the population of HIV exposed infants at national and sub-national levels in Zambia.

Methods: The study recruited a cross-sectional nationally representative sample of 8,042 caregiverbaby pairs in 38 high volume immunization sites in 7 towns across 3 provinces of Zambia. All mothers who brought their children below the age of one year for immunization at the study facilities were invited to participate in the study. All consenting mothers were interviewed and blood drawn from their babies for; rapid HIV antibody test to determine exposure and DNA PCR test for samples of all HIV-exposed babies to determine HIV infection.

Results: Of 8,042 recruited caregiver-baby pairs, 1,407 (17.5percent of all mothers of HIV exposed infants reported that they attended ANC visits more than two times and facility based deliveries stood at 92 percent. Exclusive breastfeeding among HIV exposed infants reduced with increase in age of infant; it was highest at 6 weeks (82\%) followed by 10 weeks (74\%), 14 weeks (74 \%) and 9 months (4\%). MTCT rates were relatively lower than what was reported before in subnational studies and stood at 4.94 percent among Penta 1 seekers, 3.08 percent among Penta 2 seekers, 2.63 percent among Penta 3 seekers and 5.15 percent among Measles vaccination seekers. The overall MTCT rate stood at 4.05 percent. About 46 percent of HIV positive babies were male compared to 54 percent females. Babies of mothers below the age of 25 years accounted almost half $(47 \%)$ of all HIV infected babies in the study. Reported exclusive breastfeeding among HIV positive babies was 79 percent for Penta 1 seekers, 78 percent for Penta 2 seekers and 100 percent for Penta 3 seekers. Conclusion: The study succeeded in estimating the MTCT rates using infant testing in immunization services, thereby demonstrating that it is feasible to use routine infant testing in immunization services as a strategy for estimating MTCT rates among the population of HIV-exposed infants in Zambia.

Full Text

Due to technical limitations, full-text HTML conversion of this manuscript could not be completed. 
However, the manuscript can be downloaded and accessed as a PDF.

\section{Figures}

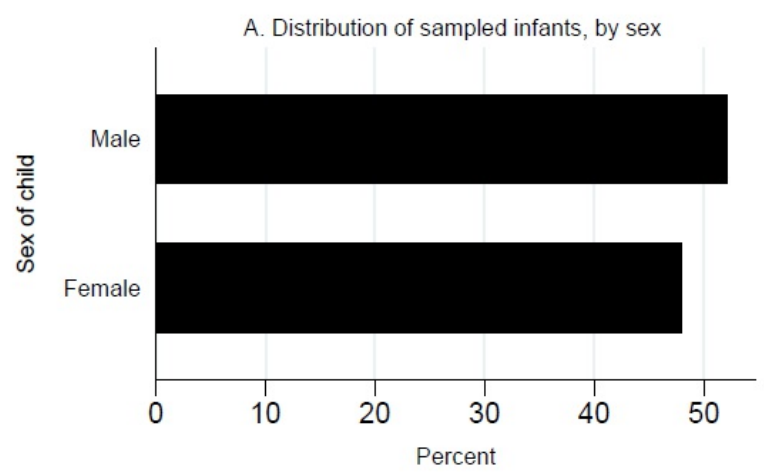

C. Distribution of infants by vaccination type

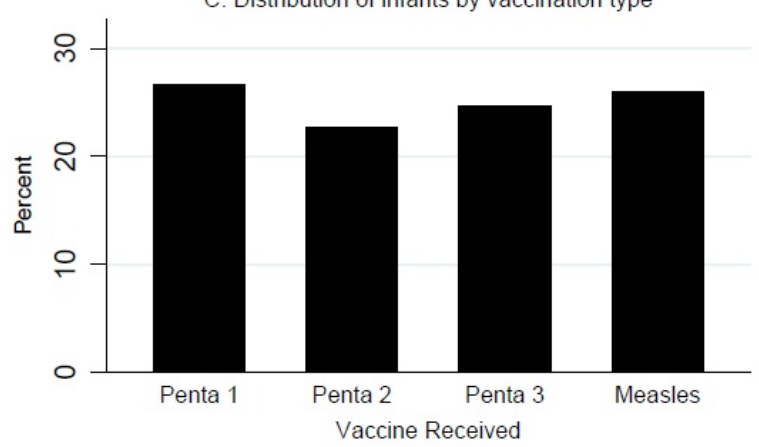

B. HIV exposed infants, by sex

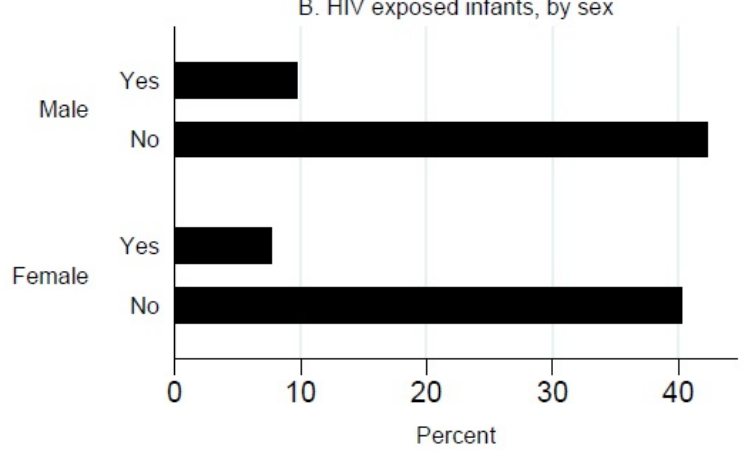

D. Infant HIV exposure rate, by vaccine type and sex

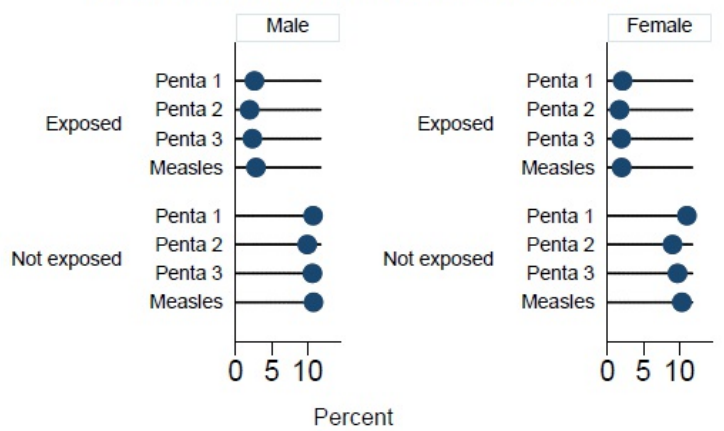

Figure 1

Infant Sex, HIV Exposure and Vaccination Type. Panel A and B of the figure shows the distribution, in percentage, of sampled infants and HIV exposure by sex. Panel C shows the infant distribution by vaccination type while Panel D shows the infant HIV exposure rate disaggregated by vaccine type and sex. 

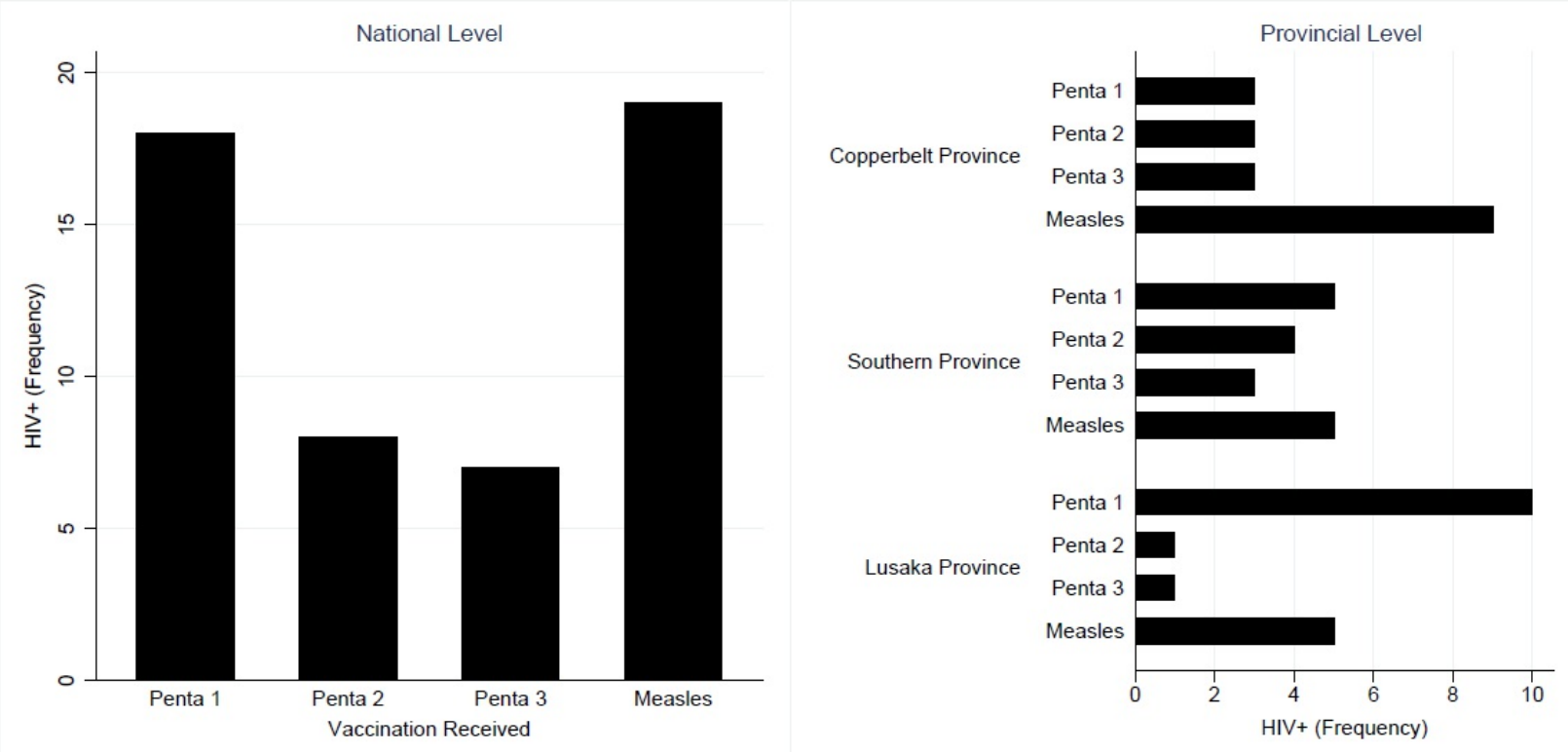

Figure 2

Number of HIV positive babies by district and infant age/immunization type. The figure shows the number of HIV positive babies by immunization type tested using DNA PCR. The distribution is illustrated at both national level and disaggregated by province. 